\title{
«Mäuse sind gute Modelle»
}

\section{Daniel Lüthi}

Freier Journalist und Fotograf, Medientrainer, Bern

1 Gollwitzer ES et al.

Lung microbiota promotes tolerance to allergens
Dies ist eine Geschichte von Mäusen und Menschen. Und von einer jungen Frau, die in ihrem Leben schon einiges erreicht hat. Erst gerade ist sie für ihre Arbeit mit dem Pfizer Forschungspreis ausgezeichnet worden. «Ein schönes i-Tüpflein», sagt Eva Gollwitzer dazu. «Der eigentliche Lohn für meine Arbeit ist es, meine Erkenntnisse unter eigenem Namen publizieren zu können» [1].

\section{Blumen, Bäume - und Bakterien}

Gollwitzer ist Biologin, arbeitet eigentlich aber für die Medizin. «Das Klischee eines Biologen, der sich mit
Birkenstock-Schuhen in Feldern und Wäldern bewegt, verkörpere ich definitiv nicht», hält sie fest. "Von Beginn weg war mir klar, dass ich nicht Käfer bestimmen will.» Während des Studiums habe sie sich zwar auch mit Blumen und Bäumen beschäftigt, ihre Bachelor- und Masterarbeit schrieb sie jedoch bereits im Themenbereich Virologie. «Es ging um die MedikamentenResistenzen von HI-Viren, und mich interessierte, wie unser Immunsystem, unsere körperliche Abwehr, gegen Eindringlinge funktioniert. Es ging also schon damals um Mechanismen.»

Darum geht es ihr heute noch. Allerdings arbeitet sie jetzt vor allem mit Bakterien. Und mit Mäusen. Kon-

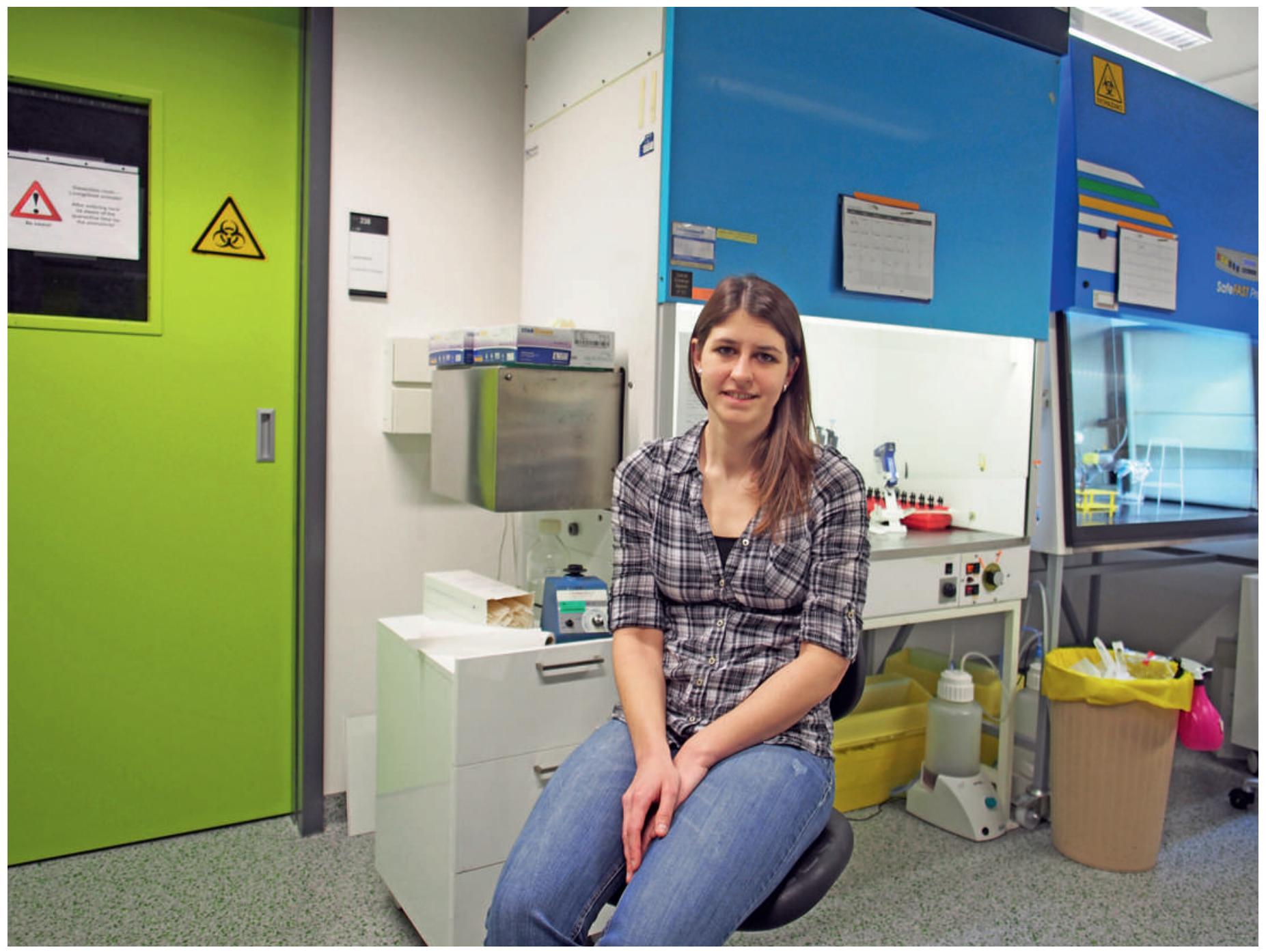


kret: «Mit der Pipette geben wir unseren Mäusen während einiger Wochen Allergene in die Nase - ein Extrakt von Hausstaub-Milben zum Beispiel, wie wir sie überall in unserer Umgebung finden.» Die Mäuse entwickeln dann, ähnlich wie Menschen, Allergien. «Dabei reagieren junge Mäuse stärker als ältere. Wir wollen wissen, warum, und was in der Lunge passiert. Eine entscheidende Rolle spielen die regulatorischen T-Zellen. Diese werden erst nach den ersten zwei Wochen im Leben der Mäuse induziert, und zwar von den Bakterien, die unseren Körper besiedeln. Sie sind dazu da, unsere Immun-Antwort herunterzufahren.» Gollwitzer übertrug diese T-Zellen von erwachsenen Mäusen auf neugeborene - die allergische Reaktion gegen Hausstaub-Milben wurde unterdrückt. Mit anderen Worten: Die ersten paar Monate nach der Geburt entscheiden darüber, ob Menschen Asthma bekommen.

\section{Pharmaindustrie und Unabhängigkeit}

Und was könnte der Nutzen dieser Erkenntnis sein? Der Pharmakonzern Pfizer schreibt dazu: «In Zukunft könnte man mit einem «Bakterien-Cocktail` den Babykörper auf natürliche Weise anregen, schützende T-reg-Zellen zu produzieren. Das könnte schon vor Ausbruch von Asthma davor bewahren - ähnlich wie eine Impfung.»

Der Pharmakonzern, der jetzt Preise ausschreibt und Arbeiten sponsert, könnte dereinst dank solcher Forschungsresultate vielleicht Geld verdienen. Kann das Forscherinnen wie Eva Gollwitzer in eine Abhängigkeit führen? «Mit meiner Arbeit bin ich in keinem Konflikt», sagt sie, "und eine gewisse Freiheit will ich in jedem Fall behalten - ich will selber entscheiden können, in welche Richtung meine Forschung gehen soll.» Das sei Teil ihres Ehrgeizes, zu dem sie ohne Wenn und Aber steht: «Ich will die Dinge gut machen, will meine Erkenntnisse publizieren - um damit unter anderem neue Gelder für unsere Forschung zu generieren.»

Die 15000 Franken Preisgeld, die sie persönlich erhalten hat, liegen erst mal auf einem privaten Konto, ihr Verwendungszweck ist noch nicht klar. Materielle Ansprüche oder Träume hat Eva Gollwitzer kaum. «Vielleicht wird mir das Geld erlauben, etwas länger in Lausanne bleiben zu können, denn hier gefällt es mir gut.»

\section{Mäuse und Menschen}

Das hat vor allem mit der Arbeit zu tun, denn diese bestimmt das Leben der jungen Frau weitgehend. Es komme schon vor, sagt sie, dass sie sieben Tage in einer Woche arbeite, vor einer Publikation beispielsweise. "Oder weil ich zu unseren Mäusen schauen muss.»

\section{Eva Gollwitzer}

Dr. Eva Gollwitzer, PhD, wurde 1986 in Fürth (Bayern, Deutschland) geboren, wo sie auch die Schulen besuchte. An der nahen Universität Erlangen-Nürnberg machte sie ihren Bachelor und Master in Biologie, dann kam sie als Doktorandin nach Lausanne, wo sie seit 2010 für die Universität und das CHUV arbeitet, seit letztem Jahr als PostDoktorandin. Dieses Jahr erhielt sie -

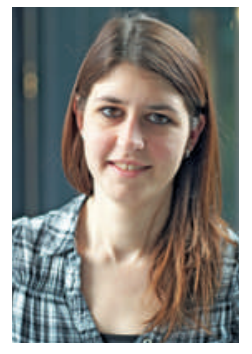
zusammen mit anderen jungen Forscherinnen und Forschern - den Pfizer Forschungspreis. Eva Gollwitzer lebt mit ihrem Partner in Lausanne.

«Unsere Mäuse` tönt fast ein wenig zärtlich, als ob es um ein liebes Haustier ginge. Nein, es sei natürlich schon ein anderer Bezug, sagt Gollwitzer, aber: «Wir verbringen viel Zeit mit unseren Tieren, müssen jeden Tag nachschauen, ob es ihnen gut geht. Denn Mäuse sind kein Material, es sind Lebewesen. Aber in unserer Forschung kommen wir ohne sie nicht aus. Sie helfen uns, gewisse Mechanismen zu entdecken. Mäuse sind gute Modelle.»

\section{«Von Beginn weg war mir klar, dass ich nicht Käfer bestimmen will.»}

Vor allem dann, wenn es darum geht, die Grundlagen für neue Medikamente zu schaffen, Krankheiten zu bekämpfen oder ihnen vorzubeugen. Mäuse im Dienste der Menschen also. «Für die Entwicklung eines Lippenstiftes oder einer Antifalten-Crème würde ich unsere Mäuse nicht zur Verfügung stellen", relativiert Gollwitzer. «Aber Allergien nehmen zu, und um sie zu bekämpfen, müssen wir verstehen, warum. Klar ist: Wir brauchen Bakterien.» Das ist keine neue Erkenntnis. «Stimmt», sagt Gollwitzer, «dass ein Kind auch mal im Dreck spielen soll, ist eine alte Weisheit. Bloss wussten wir bisher nicht genau, wie dieser Zusammenhang wirklich funktioniert.»

Das Gebiet 'Allergien und Asthma' sei noch weit offen, gleichzeitig arbeite sie in einer Nische. «Das Thema ist interessant und betrifft viele Leute, so richtig etwas dagegen machen kann man allerdings nach wie vor nicht.» Bekannt sei dies: «Wenn wir geboren werden, sind wir steril. Nachher werden wir von Bakterien kolonisiert. In den ersten drei Lebensjahren bildet sich unser Mikrobiom. Wir haben festgestellt, dass wir diese Bakterien, diese regulatorischen T-Zellen für unsere Gesundheit brauchen.» Ein allzu sauberes, ja steriles Umfeld sei also nicht gesund. Und: «Antibiotika, die zu früh verabreicht werden, sind es auch nicht zwingend.» 


\section{Forschung und Medizin}

Warum ist Eva Gollwitzer nicht Ärztin geworden? «Den direkten Kontakt mit den Patienten und diese Verantwortung wollte ich nicht», antwortet sie. «Lieber erarbeite ich Grundlagen, wirke ich indirekt in die Medizin hinein.»

Etwas distanziert wirkt sie bisweilen, aber menschenscheu keineswegs. Das Arbeiten im Team sei ihr wichtig, sagt sie. «In gewissen Labors werden die Leute gegeneinander eingesetzt, um die Sache zu beschleunigen. Das kann zu narzisstischem Ehrgeiz führen. In diesem Labor arbeiten wir lieber miteinander.»

\section{«Mäuse sind kein Material.»}

Eine Art Wettrennen sei die Forschung schon auch, aber schneller sein als andere könne man erst recht im Team. Und dazu gehören für die junge Forscherin immer wieder erfahrene Ärztinnen und Ärzte, Pneumologen oder Neonatologen zum Beispiel. «Eine gute Interaktion ist wichtig", erklärt sie. "Forscher brauchen Ärzte - und umgekehrt.»

Wichtig sei ihr auch die Vielseitigkeit, die ihr der Alltag biete: der Wechsel zwischen Praxis und Theorie, zwischen der Pipette und dem Mikroskop, dem toten Computer und den lebenden Mäusen. Zwischen der Universität und dem Spital, der Biologie und der Medizin. «Zwischen Nachdenken und Ausprobieren.»

\section{Arbeit und Familie}

Haben da Gedanken an eine eigene Familie noch Platz? "Auf jeden Fall», antwortet Eva Gollwitzer ohne zu zögern. Ihre Arbeit erlaube ihr viel Flexibilität, am Computer arbeiten könne sie auch zu Hause. «Es ist alles eine Frage der Organisation. Und es muss bei einem Paar ja nicht jeden Tag der Gleiche zurückstecken.» Als Ärztin wäre es wohl schwieriger, ein solches Leben zu führen, sinniert sie.

Und was braucht diese junge Forscherin in ihrem Leben sonst noch, ausser ihrer Arbeit und den Erfolgserlebnissen, die damit verbunden sind? "Ab und zu einen Tag in den Bergen zum Beispiel», sagt sie bescheiden, Zeit mit ihrem Partner, der Computer-Ingenieur ist, und mit ihren Freunden.

Mitte Jahr wird Eva Gollwitzer dreissig. Erst dreissig, ist einer angesichts ihres Curriculums zu denken geneigt, angesichts der Tatsache auch, dass diese junge Dame bereits angehende Doktoranden betreut und anleitet. Was wünscht sie sich zum runden Geburtstag? Sie überlegt nur kurz - und antwortet: «Dass es so gut weiterläuft, wie es angefangen hat.»

\section{Die nächste Begegnung mit ...}

Am Ende jeden Montats stellt die Schweizerische Ärztezeitung eine Persönlichkeit vor, die sich im Gesundheitswesen engagiert. Im Mai schildert Daniel Lüthi seine Begegnung mit Dr. Jörg Peltzer, Chefarzt Chirurgie in Delémont und Förderer der Unfallchirurgie in Äthiopien. 